\title{
Multiple Oscillators Provide Metastability in Rhythm Generation
}

\author{
Hong-Shiu Chang, Kevin Staras, and Michael P. Gilbey \\ Autonomic Neuroscience Institute, Department of Physiology, Royal Free and University College Medical School, \\ University College London, London NW3 2PF, United Kingdom
}

\begin{abstract}
Biological rhythms such as cardiac and circadian rhythms arise from activity of multiple oscillators with dispersed intrinsic frequencies. It has been proposed that a stable population rhythm, fundamental to normal physiological processes, can be achieved in these systems by synchronization, through mutual entrainment, of individual oscillators. Mutual entrainment, however, is unlikely to be the mechanism underlying the generation of a stable rhythm in a population of multiple weakly coupled or uncoupled oscillators. We have recently identified such a population that is involved in the sympathetic regulation of vascular tone in a thermoregulatory circulation. In this paper, we investigate the stability of the output rhythm of these sympathetic oscillators by subjecting the system to a periodic driving force (the lung inflation cycle-related activity). We show that a population rhythm coupled to the drive can remain stable over a
\end{abstract}

much wider driving frequency range compared with that of any one of its constituent oscillators. This population rhythmicity still exists despite the fact that the dominant frequencies of individual oscillators are not necessarily 1:1 frequency-locked to the drive. We provide evidence to show that this population metastability is achieved through linear and nonlinear dynamic interactions between the driving force and single sympathetic oscillators. Our study suggests that the generation of a stable population rhythm can exist even in the absence of mutual entrainment of its constituents, and this allows the population to generate a stable and flexible patterned response.

Key words: postganglionic sympathetic neuron; neural oscillator; synchronization; entrainment; nonlinear dynamics; blood vessel; in vivo; Sprague Dawley rat
In vertebrates, the genesis of essential biological rhythms, such as cardiac (DeHaan and Hirakow, 1972; Sano et al., 1978) and circadian (Welsh et al., 1995; Herzog et al., 1998) rhythms, involves oscillatory activity generated by multiple oscillators. There is considerable demand for stability in these rhythms, yet the population is often composed of oscillators with dispersed intrinsic frequencies $\left(\mathrm{f}_{\mathrm{INT}} \mathrm{s}\right)$. This raises the issue of how the constituent oscillators interact so that a stable population rhythm emerges. Mutual entrainment (i.e., frequency-lock) (Winfree, 1980) of individual oscillators, in the absence of an external driving force, has been proposed as a mechanism of synchronization to generate, for example, cardiac (Jalife, 1984; Michaels et al., 1986) and circadian (Liu et al., 1997) rhythms. An alternative organization has been established in a multiple oscillator population influencing vascular conductance (Johnson and Gilbey, 1994, 1996; Chang et al., 1999b), where the individual oscillators are weakly coupled or uncoupled (Chang et al., 1999b). In this type of system, individual oscillators can still be entrained to a common periodic drive (Winfree, 1980; Chang et al., 1999b). However, such a mechanism is unlikely to be sufficient for producing a stable population rhythm, coupled to a driving force, over a very large frequency range, because for biological oscillators, 1:1 entrainment frequently operates only when the driving frequency is close to the intrinsic frequency of the driven oscillator (Glass and Mackey, 1988). The demand for stability of

\footnotetext{
Received Oct. 21, 1999; revised April 6, 2000; accepted April 11, 2000.

H.-S.C. was supported by Chang Gung Memorial Hospital. This work and K.S. were supported by Wellcome Grant 05115 .

H.-S.C. and K.S. contributed equally to this work.

Correspondence should be addressed to Dr. Michael P. Gilbey, Autonomic Neuroscience Institute, Department of Physiology, Royal Free University College Medical School, Royal Free Campus, UCL, Rowland Hill Street, London NW3 2PF, UK. E-mail: mpg@rfhsm.ac.uk.

Copyright (C) 2000 Society for Neuroscience $\quad 0270-6474 / 00 / 205135-09 \$ 15.00 / 0$
}

population rhythms across a wide driving frequency range may be particularly important for coordinating activity of the sympathetic nervous system and other rhythm-generating circuits such as the respiratory network (Gilbey and Spyer, 1997).

In this paper we investigated the underlying coupling dynamics that may contribute to stability and flexibility in a multiple oscillator network of this type. We did this by examining the frequency-dependent characteristics of the oscillator population in response to an external periodic input: lung inflation cycle (LIC)-related activity (Lipski et al., 1977). The oscillator activity can be conveniently monitored at single and population levels by recording the discharge behavior of postganglionic neurons (PGNs). Previous work has established that individual PGNs (sample size $>100$ ) exhibit a distinct intrinsic rhythm in their discharges that has a frequency $\left(f_{\text {INT }}\right.$ ) within a range of $\sim 0.4-1.2$ $\mathrm{Hz}$ (Johnson and Gilbey, 1996; Chang et al., 1999b; and our unpublished observations). We provide evidence to show that, even outside the intrinsic frequency range of individual oscillators, a periodic input across a wide frequency range can produce a stable population rhythm. We demonstrate that a population rhythm emerged, even when individual oscillators did not exhibit 1:1 entrainment. Over a narrow LIC frequency range, near their intrinsic frequency, individual oscillators showed stable coupling and high coherence with LIC-related activity. Outside this range, coherence dropped precipitously at single oscillator level. In comparison, at the population level the 1:1 frequency response range was extended. We provide evidence to show that this feature arises from linear and nonlinear dynamic LIC-PGN interactions at the single oscillator level, which with their intrinsic frequency spread confers the population with metastability for rhythm genesis. In the sympathetic system, this metastability of synchronized neural activity is likely to be important for provid- 
ing graded control of blood flow at the vascular end organ. In more general terms, complexity of single oscillator behavior may provide a mechanism for producing stable and flexible output in various rhythmic activities generated by multiple oscillators.

Part of this work has been published previously as an abstract (Chang et al., 1999a).

\section{MATERIALS AND METHODS}

General preparation and maintenance. All experiments were performed in accordance with the Animal (Scientific Procedures) Act, UK, 1986. Eighteen male Sprague Dawley rats $(235-350 \mathrm{gm})$ were anesthetized with pentobarbitone $(60 \mathrm{mg} / \mathrm{kg}$, i.p. $)$, supplemented with $\alpha$-chloralose (5-10 mg, i.v.) when required. An adequate anesthetic level was indicated by the stability of blood pressure (BP) and respiratory activity, monitored by diaphragm EMG, and the absence of both corneal and paw-pinch withdrawal reflexes. The femoral artery and vein were cannulated for monitoring BP and infusing drugs, respectively. The urinary bladder was cannulated to ensure an unobstructed urinary flow. The esophageal temperature was monitored and maintained at $36.5-37^{\circ} \mathrm{C}$ using a heating blanket. LICs were recorded using tracheal pressure (TP) waves. Using hyperoxia induced by artificial ventilation with $\sim 95 \%$ oxygen, all experiments were performed during central apnea to minimize influence from central respiratory activity (Chang et al., 1999b). Quiescence of respiratory activity was indicated by the absence of diaphragm EMG activity. Samples of arterial blood $(70 \mu \mathrm{l})$ were taken regularly $(\sim 20-40 \mathrm{~min})$ for gas and $\mathrm{pH}$ analyses.

Neural activity recording. The caudal ventral artery (CVA) of the tail was exposed, and the overlying connective tissue was removed. The CVA was then positioned in a bath filled with Krebs' solution. Using glass microelectrodes (internal diameter of the tip, 20-100 $\mu \mathrm{m}$ ), single PGNs ( $n=21$ from 12 animals) were recorded focally from the surface of the CVA, and 10 of these were recorded in pairs (five pairs). PGN activity was monitored on a digital oscilloscope, and activity from single PGNs was identified by a consistent spike waveform and amplitude. For detail of the focal recording technique, see Johnson and Gilbey $(1994,1996)$ and Chang et al. (1999b). Population PGN activity ( $n=6$ from six animals) was recorded from the ventral collector nerve (VCN), which contains $\sim 80 \%$ of the PGN axons innervating the CVA (Sittiracha et al., 1987). The VCN was isolated from the tail, cut, desheathed, and immersed in a bath of paraffin oil. The distal end was crushed. Biplolar platinum hook electrodes were used to record nerve activity. In wholenerve experiments, the cauda equina was cut at L5 level to remove contamination of somatic motor activity (Sittiracha et al., 1987; Smith et al., 1998). A previous study has established that using this preparation, activity recorded from the VCN is sympathetic in nature (Chang et al., 1999b).

Data collection. Neural activity was processed as described previously (Chang et al., 1999b). Briefly, PGN and diaphragm EMG activity were amplified and filtered (bandpass, $300-1000 \mathrm{~Hz}$ ). VCN and diaphragm activity were rectified and smoothed (time constant, $100 \mathrm{msec}$ ). Data of 5 and 8 min length were recorded for single PGN and population PGN experiments under each ventilation condition (see Experimental protocols), respectively. Data were stored on tape for off-line analysis. In addition, BP, TP, single PGN activity, and smoothed VCN and diaphragm activity were converted to digital signals and sent to computer for analysis (sampling rate: $13.3 \mathrm{kHz}$ for single PGN activity; $100 \mathrm{~Hz}$ for smoothed VCN and diaphragm activity). Using spike processors and a pulse-generating interface, transistor-to-transistor logic pulses were generated from the action potentials of single PGNs and the inflation phase of TP. These pulses were used to construct autocorrelograms and LIC $\rightarrow$ PGN cross-correlograms (Chang et al., 1999b).

Experimental protocols. $f_{\mathrm{INT}} \mathrm{S}$ in single and population PGN activity, was first determined during "free-run" using high LIC frequency (1.9-2.5 $\mathrm{Hz}$ ) and low tidal volume (1-1.2 ml) to "unlock" LICs and PGN activity. The frequency-dependent responses were examined by varying LIC frequency $\left(f_{\text {LIC }}\right)$ while maintaining a constant high tidal volume (2-2.5 $\mathrm{ml})$. Initially, $f_{\text {LIC }}$ was adjusted to near $f_{\text {INT }}$ of PGN activity, and stable 1:1 entrainment was established. To demonstrate that this was not a chance phenomenon, $f_{\text {LIC }}$ was tested for two or three separate steps $(0.1$ $\mathrm{Hz}$ ) away from $f_{\text {INT }}$ to confirm that 1:1 entrainment did not occur just at one single $f_{\text {LIC }}$. After this, $f_{\text {LIC }}$ was increased in 0.2 or $0.3 \mathrm{~Hz}$ steps up to $\sim 1.5-1.8 \mathrm{~Hz}$. Some single PGNs $(n=8)$ were not carried through the whole frequency range of $f_{\text {LIC }}$ because of difficulties in maintaining stable recording conditions. To test whether hypocapnia affected the discharge patterns of PGN activity, while maintaining a central apneic state, $\mathrm{CO}_{2}$ $(<5 \%)$ was given to the rats in half of the whole nerve experiments when hypocapnia developed during periods of high frequency and high tidal volume ventilation. The amount of $\mathrm{CO}_{2}$ given was adjusted to keep $\mathrm{Pa}_{\mathrm{CO}_{2}}$ close to the normal physiological range $(\sim 35-40 \mathrm{mmHg})$, and the discharge behavior of VCN activity from experiments in which $\mathrm{PaCO}_{2}$ was and was not clamped was compared.

Data analysis. Spectral analysis was used to determine the frequencies of rhythmical components in neural activity and LIC. For single unit experiments, spike trains of PGN activity were first transformed to event count time series (counts every $10 \mathrm{msec}$ ) before spectral analysis (Rosenberg et al., 1989). The time series of PGN event counts, VCN activity, and TP waves were divided into half-overlapping blocks (length, 20.48 sec). The linear trend in each block was removed and windowed by a Hanning taper, followed by 2048-point Fast Fourier Transformation (Bendat and Piersol, 1986). The overall autospectrum was generated by averaging the autospectrum from each block. In all the autospectra, maximal power density was normalized to 1 for a direct comparison of dominant frequencies across different activities. The spectral resolution was $0.049 \mathrm{~Hz} /$ bin. The dominant rhythm of single PGN frequency was first confirmed by the presence of rhythmicity in the self-triggered autocorrelogram (Perkel et al., 1967a), and the exact frequency was determined from the autospectrum. For population PGN activity, the dominant frequency was defined as the frequency of the spectral peak associated with highest power density.

The coupling strength at a particular frequency between paired activities (single PGN $\rightarrow$ single PGN, LIC $\rightarrow$ single PGN, LIC $\rightarrow$ population PGN) was evaluated by squared coherence (abbreviated as coherence) (Bendat and Piersol, 1986). The coherence is a bound parameter with 1 indicating perfect correlation and zero indicating no correlation. Data of frequency and coherence are displayed as median and first to approximately third interquartile range. The $\mathrm{LIC} \rightarrow \mathrm{PGN}$ cross correlogram (Perkel et al., 1967b), triggered by the inflation phase of TP waves, was used to measure the phase difference between them. The temporal stability of this phase difference was evaluated by LIC $\rightarrow$ PGN correlation raster plots (CRP) (Chang et al., 1999b). Similar to the crosscorrelogram, the CRP demonstrates the phase relationship between the triggers (LICs) and the dependent event activity (PGN activity). The dependent events are plotted according to their peri-trigger lags. This process is reset every time another trigger is added. However, instead of accumulating events in bins over the analysis period (as for a crosscorrelogram), for CRPs the relationship between triggers and dependent events is plotted for each trigger. This reveals temporal stability and provides an assessment of phase difference. The presence of dense vertical bands in the CRP would suggest that constancy of phase difference is a stationary feature across time. However, it does not necessarily indicate that only one single phase difference exists. For example, phase difference under conditions of 2:1 entrainment alternates between two stable values from cycle to cycle, and this would also produce vertical striations in the CRP. To evaluate the distribution of phase differences across a whole cycle of the PGN dominant rhythm, the CRP was reordered by the first post-LIC interevent intervals [termed reordered correlation raster plot (RCRP)]. This enables PGN events with distinct stable phase differences to LIC to cluster in different dense vertical bands. In addition, for uncorrelated LICs and single PGN activity, RCRP also provides information about the randomness of phase shift between them because in this case their phase difference will be any value in the cycle of the PGN rhythm and produce a slanted band covering the whole cycle in the RCRP. Complementary to CRP, RCRP provides further information regarding the temporal characteristics of phase difference (Chang et al., 1999b). The CRP and RCRP were divided into small quadrats ( $0.05 \mathrm{sec}$ by 10 trigger events), and the PGN events in each quadrat were counted as a measure of the event density. The event density of these raster plots was displayed using a gray scale.

The changes of parameters across the range of $f_{\text {LIC }}$ tested were evaluated by linear regression. A deviation of the slope of the regression line from zero was considered to be significant if $p<0.05$ (Student's $t$ test) (Glantz, 1996).

\section{RESULTS}

\section{Condition of animals}

The scatter plots in Figure 1 summarize the changes of physiological parameters $\left(\mathrm{BP}, \mathrm{Pa}_{\mathrm{O}}, \mathrm{Pa}_{\mathrm{CO}_{2}}, \mathrm{pH}\right)$ in response to $f_{\mathrm{LIC}}$ variations. The significance of covariation between these param- 
A Whole nerve experiments

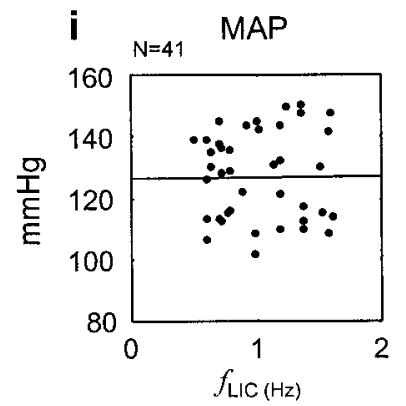

B Single PGN experiments

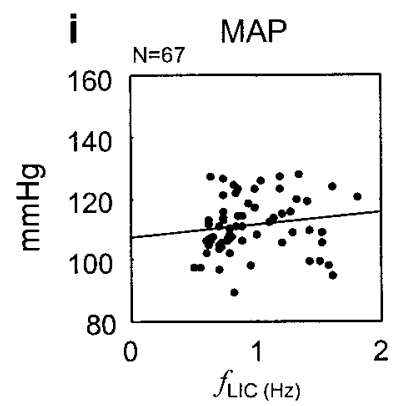

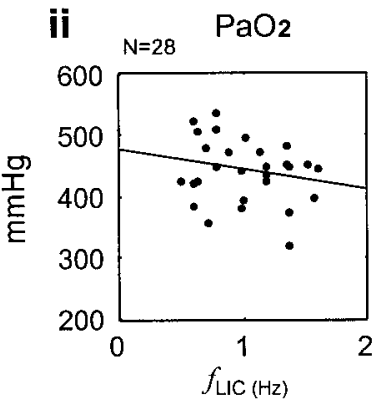
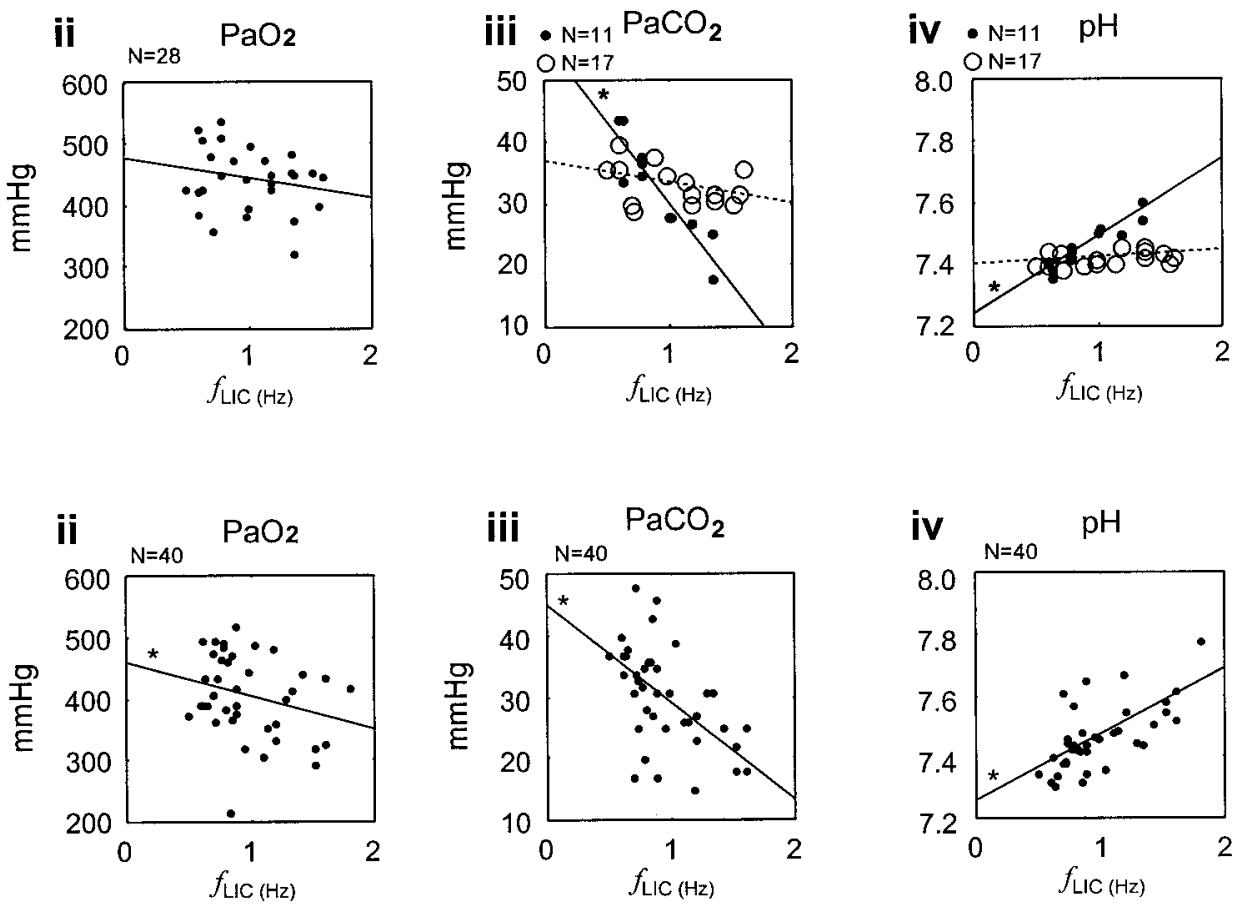

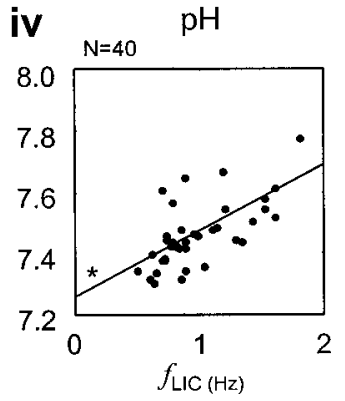

Figure 1. Scatter plots of physiological parameters across different $f_{\mathrm{LIC}} \mathrm{s}$. Significant change of the parameters, evaluated by whether the slope of the regression line deviates from zero, is indicated by an asterisk ( $t$ test, $p<0.05)$. $A$, Whole nerve experiments. $i$, $i i$, No significant change across different $f_{\mathrm{LIC}} \mathrm{S}$ is present in the mean arterial pressure $(M A P)$ and $\mathrm{Pa}_{\mathrm{O}_{2}}$ as $f_{\mathrm{LIC}}$ varies. iii, iv, When $f_{\mathrm{LIC}}$ is high and no $\mathrm{CO}_{2}$ is added ( filled circles, solid line), respiratory alkalosis develops with low $\mathrm{Pa}_{\mathrm{CO}_{2}}$ and high $\mathrm{pH}$ ( $t$ test for zero slope, $p<0.001$ for both). The $\mathrm{Pa}_{\mathrm{CO}_{2}}$ and pH do not change significantly across different $f_{\mathrm{LIC}} \mathrm{s}$ in experiments in which $\mathrm{CO}_{2}$ is added (open circles, dotted line). $B$, Single PGN experiments. $i$, No significant change across different $f_{\mathrm{LIC}} \mathrm{S}$ is present in MAP as $f_{\mathrm{LIC}}$ is changed. $i i$, A borderline negative trend ( $t$ test for zero slope, $\left.p=0.048\right)$ in $\mathrm{Pa}_{\mathrm{O}_{2}}$ is observed when $f_{\mathrm{LIC}}$ is increased. $i i i$, $i v$, Respiratory alkalosis develops with low $\mathrm{Pa}_{\mathrm{CO}_{2}}$ and high $\mathrm{pH}$ ( $t$ test for zero slope, $p<0.001$ for both) during periods of high $f_{\mathrm{LIC}}$.

eters and $f_{\text {LIC }}$ was assessed by linear regression. There was no significant influence of $f_{\text {LIC }}$ on BP (Fig. $1 \mathrm{Ai}, 1 \mathrm{Bi}$ ), which is consistent with previous studies in rats (Marshall, 1994). No significant change of $\mathrm{Pa}_{\mathrm{O}_{2}}$ was found in the whole-nerve experiments (Fig. 1 Aii), although a borderline negative trend was observed in the single PGN experiments ( $t$ test for zero slope of the regression line, $p=0.048$ ) (Fig. $1 \mathrm{Bii}$ ). When $f_{\text {LIC }}$ was high, respiratory alkalosis developed as a result of pulmonary alveolar hyperventilation in experiments in which no $\mathrm{CO}_{2}$ was added. Under such conditions, $\mathrm{Pa}_{\mathrm{CO}_{2}}$ dropped and $\mathrm{pH}$ increased (Fig. 1Aiii, Aiv, Biii, Biv, filled circles). Respiratory alkalosis was prevented in half the whole nerve experiments by adding $\mathrm{CO}_{2}$ (Fig. 1Aiii, Aiv, open circles). The discharge behavior of $\mathrm{VCN}$ activity was not different under the two conditions (see below).

\section{Behavior of single PGN or PGN pairs}

Figure $2 A$ shows a typical example of single PGN neurograms and TP waves (LICs) and their associated autospectra from an experiment in which two PGNs were recorded simultaneously. Under free-run conditions, each single PGN exhibited a dominant $f_{\text {INT }}$ in its discharges (median frequency, $0.69 \mathrm{~Hz}$; interquartile range, $0.66-0.73 \mathrm{~Hz}$ ). In the five pairs in which two PGNs were recorded simultaneously, the $f_{\mathrm{INT}} \mathrm{S}$ of the paired PGNs were never the same $\left(f_{\text {INT }}\right.$ for PGN1: $0.78 \mathrm{~Hz}$, PGN2: $0.69 \mathrm{~Hz} ; f_{\text {LIC }}$, 2.00) (Fig. 2Ai). This is consistent with previous findings (Chang et al., 1999b) and provides good evidence that this is a multiple oscillator population, with each oscillator having a characteristic intrinsic dominant rhythm. The $\mathrm{LIC} \rightarrow \mathrm{PGN}$ coherence spectra for the same periods of data for the two units are shown in Figure 3 . Under free-run conditions, coherence at $f_{\text {LIC }}$ between LICPGN and PGN-PGN was near zero as revealed in Figure $3 A i$, and no fixed-phase relationship between LICs and PGN activity was observed as demonstrated in the cross-correlogram (Fig. 4Ai; the unit is the same as PGN1 with the same period of data in Figs. $2 A i, 3 A i$ ). The PGN events distributed uniformly in the CRP (Fig. 4 Aii) and spanned the whole cycle of the PGN dominant rhythm as revealed in the RCRP (Fig. 4Aiii), suggesting that phase differences between LIC and PGN activity changed constantly, i.e., they were asynchronous. Moving $f_{\mathrm{LIC}}$ into $f_{\mathrm{INT}}$ range led to stable $1: 1$ entrainment with high $\mathrm{LIC} \rightarrow \mathrm{PGN}$ coherence at $f_{\text {LIC }}$ $(0.60 \mathrm{~Hz})$ (Figs. 2Aii, 3Aii), and in cases in which two PGNs were recorded simultaneously, this resulted in high coherence between the two (Fig. 3Aii). The PGN activity was periodically synchronized to LICs as shown in the $\mathrm{LIC} \rightarrow$ PGN cross-correlogram (Fig. $4 B i$; the unit is the same as $P G N 1$ with the same period of data in Figs. 2Aii, 3Aii). The presence of a single $\mathrm{LIC} \rightarrow \mathrm{PGN}$ phase difference with high temporal stability was revealed by the dense vertical striations in the CRP and RCRP (Fig. 4Bii, Biii). This demonstrates that the 1:1 coupling between LICs and PGN activity became a strong and steady feature across time. LICPGN 1:1 entrainment with high coherence was retained through a range of different $f_{\text {LIC }}$ s (e.g., $0.70 \mathrm{~Hz}$ ) (Figs. 2Aiii, 3Aiii) above which 1:1 entrainment probability started to decline. The exact 1:1 frequency-lock range of different PGNs was rarely the same, presumably reflecting the spread of $f_{\mathrm{INT}} \mathrm{S}$ within the population. 
A .... PGN1 - PGN2 - TP (LIC)

$f_{\mathrm{LIC}}(\mathrm{Hz})$

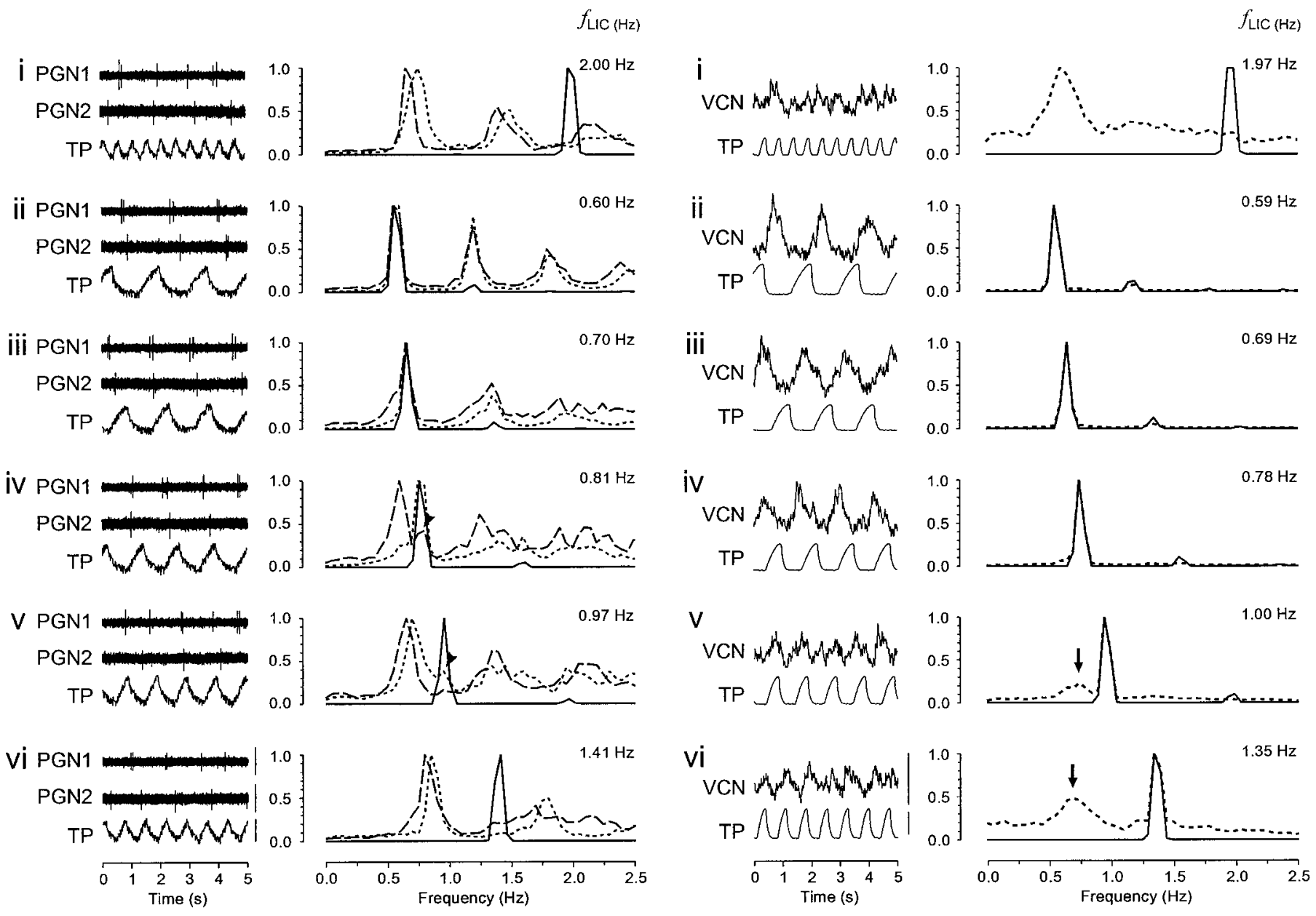

B

$$
\ldots \text { VCN }- \text { TP }(\mathrm{LIC})
$$

Figure 2. Frequency-dependent entrainment of PGN activity to LICs. A, Real time data (left panel) and superimposed autospectra (right panel) of dual recorded PGNs (dotted and dashed lines) and TP (LIC, solid line). $i$, Both neurons show an intrinsic dominant rhythm ( $f_{\text {INT }}$ for PGN1 and PGN2, 0.78 and $0.69 \mathrm{~Hz}$, respectively) under free-run conditions when $f_{\text {LIC }}$ is high $(2.00 \mathrm{~Hz})$. ii-iii, When $f_{\text {LIC }}$ is moved into $f_{\text {INT }}$ range $\left(f_{\text {LIC: }}: 0.60-0.70 \mathrm{~Hz}\right)$, stable 1:1 entrainment results. $i v$, At $f_{\text {LIC }}=0.81 \mathrm{~Hz}$, the PGN with the higher $f_{\mathrm{INT}}(P G N 1)$ still shows entrainment but the other unit (PGN2) fails to lock. $v-v i$, At higher $f_{\text {LIC }} \mathrm{S}(0.97-1.41 \mathrm{~Hz})$ both units are not entrained to LICs. The small peak at $f_{\text {LIC }}$ (arrowheads in $\left.i v, v\right)$ indicates minor LIC-related rhythmical components, suggesting relative coordination. Calibration: $25 \mu \mathrm{V}(P G N), 10 \mathrm{mmHg}(T P)$. B, Real time data (left panel) and superimposed autospectra of the PGN population ( VCN, dotted line $)$ and $T P(L I C$, solid black line $) . i$, During free-run conditions $\left(f_{\mathrm{LIC}}: 1.97 \mathrm{~Hz}\right)$, the population PGN activity reveals a broad peak (modal frequency, $0.64 \mathrm{~Hz}$ ) representing the spread of $f_{\mathrm{INT}} \mathrm{S}$ within the population. $i i-i v$, Moving $f_{\text {LIC }}$ into this range $\left(f_{\text {LIC }}: 0.59-0.78 \mathrm{~Hz}\right)$ results in a single narrow peak at $f_{\text {LIC }}$ indicating that activity of most PGNs is $1: 1$ entrained to LICs. $v-v i$, At higher ventilation frequencies $(1.00-1.35$ $\mathrm{Hz}, v-v i)$ a narrow peak at $f_{\text {LIC }}$ is still preserved although some PGNs escape 1:1 entrainment as indicated by minor peaks in the $f_{\text {INT }}$ range ( $v, v i$, arrows). Calibration: $0.5 \mu \mathrm{V}(V C N), 10 \mathrm{mmHg}(T P)$.

The phenomenon of differential frequency-lock is exemplified in Figure 2 Aiv. When $f_{\text {LIC }}$ was increased to $0.81 \mathrm{~Hz}$, although the PGN with higher, $f_{\text {INT }}$ (PGN1) was still 1:1 entrained to LICs; the other with lower $f_{\text {INT }}$ (PGN2) was not. When $f_{\text {LIC }}$ was increased further, single PGNs could not maintain 1:1 entrainment to LICs [ $f_{\text {LIC }}: 0.97 \mathrm{~Hz}$ (Fig. $2 A v$ ) and $1.41 \mathrm{~Hz}$ (Fig. 2Avi)]. However, it should be emphasized that the deviation of the dominant PGN frequency from $f_{\text {LIC }}$ did not exclude the possibility of the existence of a minor LIC-related component. This occurred especially when $f_{\text {LIC }}$ was just above the $1: 1$ entrainment frequency zone (Fig. 2Aiv, PGN2, arrowhead; Fig. 2Av, PGN1, arrowhead). This provides an explanation for those cases in which high LIC $\rightarrow$ PGN coherence at $f_{\text {LIC }}$ was still preserved despite a different dominant PGN rhythm (e.g., coherence, 0.67 for PGN2 in Fig. 3 Aiv and 0.36 for PGN1 in Fig. 3Av). This nondominant LICrelated activity arose from intermittent "attraction" of the PGN oscillator to the LICs, a phenomenon reminiscent of relative coordination (von Holst, 1939; Ayers and Selverston, 1979; Rosenblum and Turvey, 1988). In the $\mathrm{LIC} \rightarrow \mathrm{PGN}$ crosscorrelogram, it manifested itself as periodic LIC-related peaks standing out of a non-LIC modulated level [(Fig. 4Ci) the unit is the same as PGN1 with the same period of data in Figs. $2 A v$, $3 A v$ ]. This dynamic feature of LIC-PGN interaction is clearly shown in the CRP where vertical bands with varied event density across time were present in a phase-drift background (Fig. 4Cii), suggesting that PGN activity was intermittently and transiently locked to LICs. This was further supported by the RCRP in which short vertical bands (arrows) were superimposed on the cycle-spanned PGN event striations (Fig. 4Ciii). When $f_{\text {LIC }}$ was moved further away from the $f_{\text {INT }}$ range of PGNs, LIC $\rightarrow$ PGN and $\mathrm{PGN} \rightarrow \mathrm{PGN}$ coherence dropped sharply (Fig. 3Av, 3Avi), and the probability of LIC-PGN lock decreased as suggested in 


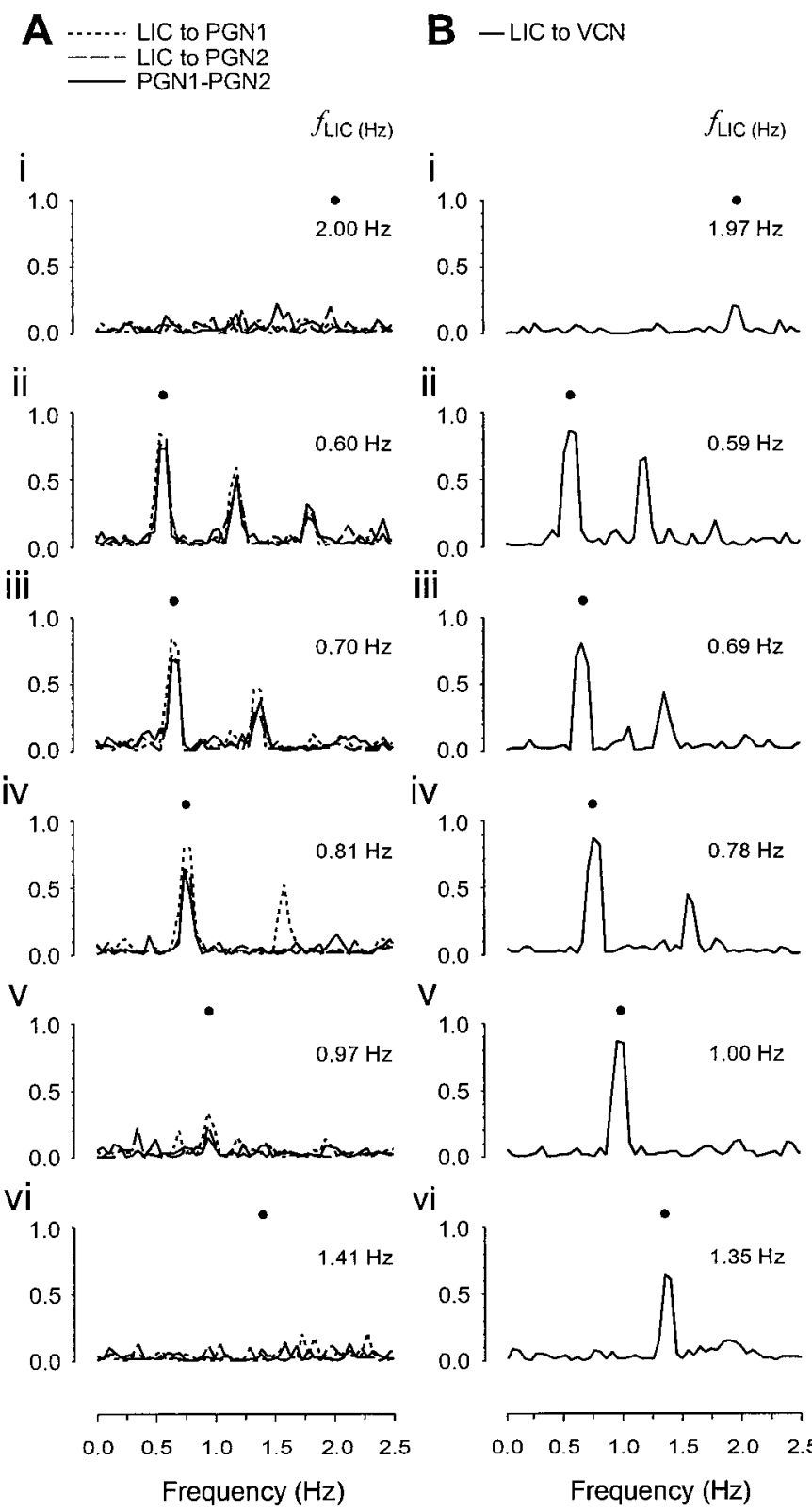

Figure 3. Frequency response of coupling strength between PGN activity and/or LICs. The strength of coupling is evaluated by the coherence spectrum. The neural and LIC activities are the same as those in Figure 2. $f_{\mathrm{LIC}} \mathrm{S}$ are indicated by filled circles. $A, \mathrm{LIC} \rightarrow \operatorname{single}$ PGN (dotted and dashed lines) and single PGN $\rightarrow$ single PGN (solid line) coherence spectra. $i$, During free-run $\left(f_{\mathrm{LIC}}: 2.00 \mathrm{~Hz}\right), \mathrm{LIC} \rightarrow \mathrm{PGN}$ and $\mathrm{PGN} \rightarrow \mathrm{PGN}$ coherence at $f_{\mathrm{LIC}}$ is minimal, indicating weak coupling between them. $i i-i i i$, When $f_{\text {LIC }}$ is moved close to the $f_{\text {INT }}$ of the PGNs $\left(f_{\text {LIC }}: 0.60-0.70 \mathrm{~Hz}\right)$, strong LIC-PGN coupling strength at $f_{\text {LIC }}$ emerges as revealed by the high coherence between them. $i v$, The strong coupling at $f_{\text {LIC }}$ is still preserved although the dominant frequency of one unit, PGN2, is different from $f_{\text {LIC }}$ when $f_{\text {LIC }}$ is increased to $0.81 \mathrm{~Hz}$ (compare with Fig. 2Aiv), a phenomenon believed to arise from relative coordination. $v-v i$, When $f_{\mathrm{LIC}}$ is increased further, the coherence and therefore the coupling strength at $f_{\text {LIC }}$ drop $\left(f_{\text {LIC }}: 0.97-1.41 \mathrm{~Hz}\right) . B$, LIC $\rightarrow$ VCN (population PGN) coherence spectra. $i$, Under free-run conditions $\left(f_{\mathrm{LIC}}: 1.97 \mathrm{~Hz}\right)$, the $\mathrm{LIC} \rightarrow \mathrm{VCN}$ coherence at $f_{\text {LIC }}$ is low, suggesting that most single PGNs are not coupled through LICs. $i i-v$, When $f_{\text {LIC }}$ is moved into the range of PGN $f_{\text {INT }}$, high coherence emerges and is maintained through a wide $f_{\text {LIC }}$ range $(0.59-$ $1.00 \mathrm{~Hz}$ ). vi, Although a moderate drop is observed, the coherence remains high although $f_{\text {LIC }}$ is above the $f_{\text {INT }}$ range $\left(f_{\text {LIC }}: 1.35 \mathrm{~Hz}\right)$.
A $2.00 \mathrm{~Hz}$ ('Free-Run')
C $0.97 \mathrm{~Hz}$ (Relative Coordination)

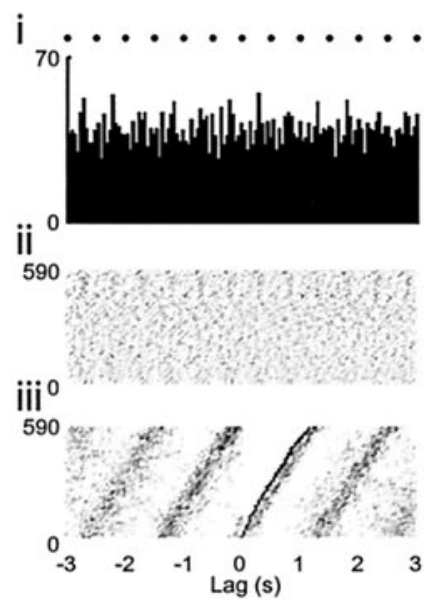

B $0.60 \mathrm{~Hz}$ (1:1 Entrainment)
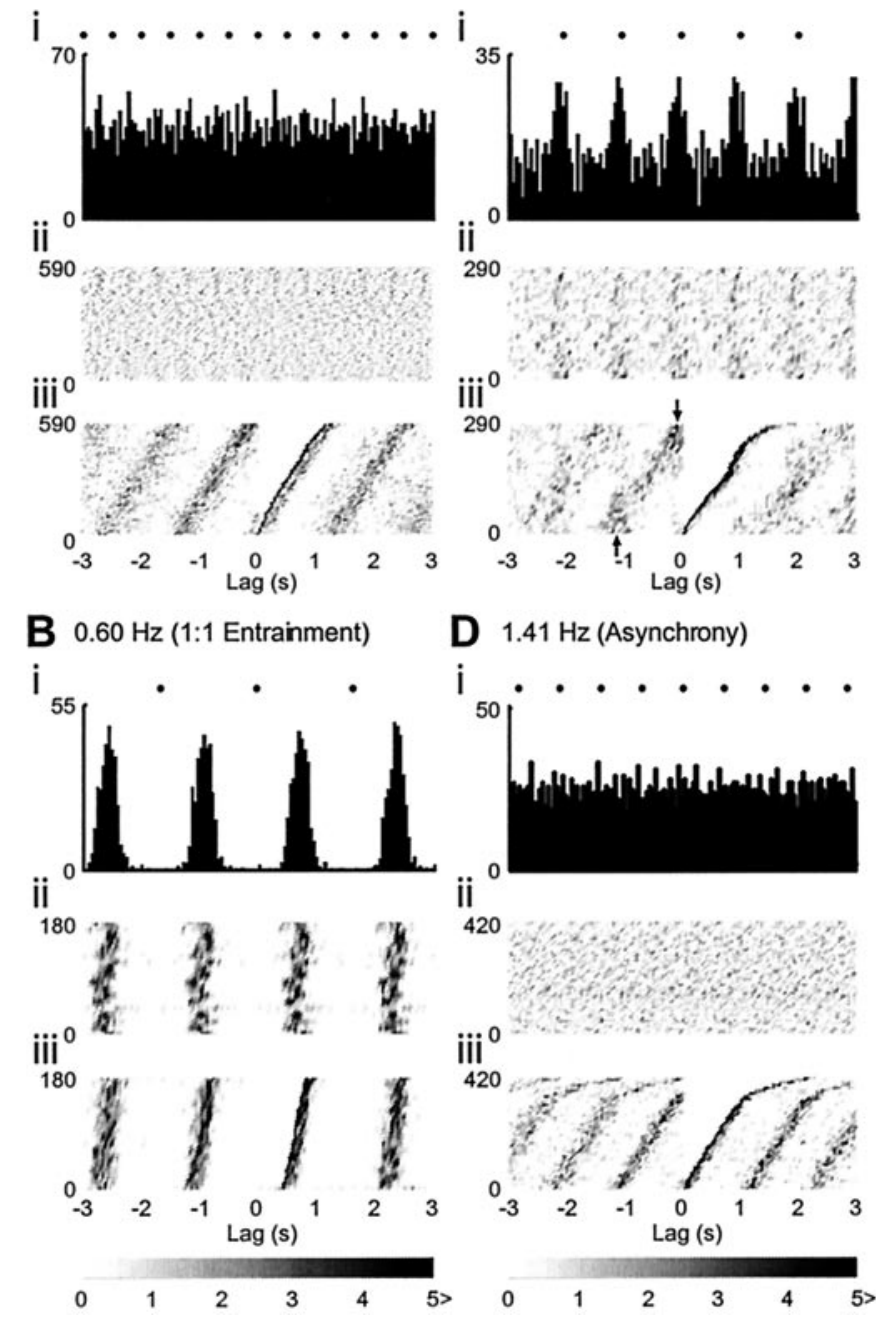

D $1.41 \mathrm{~Hz}$ (Asynchrony)

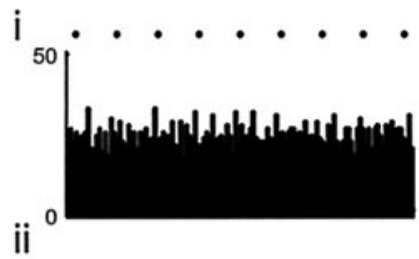

Figure 4. Phase relationships between single PGN activity and LICs. The neural and LIC activities are the same as those in Figures 2 and 3 (PGN1). The relative positions of the LIC occurrences are indicated by filled circles. $A$, Free-run. During free-run $\left(f_{\mathrm{LIC}}: 2.00 \mathrm{~Hz}\right)$, the LIC $\rightarrow$ PGN crosscorrelogram is essentially flat, suggesting noncorrelation $(i)$. Constant phase-drift across time during these periods is indicated by the uniform distribution of PGN events in CRP (ii) and their cycle-spanning pattern in RCRP (iii) $B, 1: 1$ entrainment. When $f_{\text {LIC }}$ is moved into $f_{\text {INT }}$ range $\left(f_{\text {LIC }}\right.$ : $0.60 \mathrm{~Hz}$ ), $1: 1$ entrainment emerges, and the cross-correlogram shows a pattern of rhythmical synchronization $(i)$. A stationary fixed-phase relationship between PGN activity and LICs is revealed as stable vertical bands in both CRP (ii) and RCRP (iii). $C$, Relative coordination. When $f_{\text {LIC }}$ was moved away from $f_{\text {INT }}(0.97 \mathrm{~Hz})$, intermittent periods of phase-lock occurred, and it was manifested as LIC-related periodic peaks superimposed on a background level in the cross-correlogram $(i)$. The dynamic nature of this phase-lock is indicated by intermittent vertical bands with variable density in CRP (ii) and vertical bands (arrows) superimposed on cyclespanning bands in RCRP (iii). $D$, Asynchrony. When $f_{\text {LIC }}$ is moved farther away from PGN $f_{\text {INT }}\left(f_{\text {LIC }}: 1.41 \mathrm{~Hz}\right)$, a pattern similar to that during free-run (compare with $A$ ), suggestive of complete asynchrony, appears. ( $i$, cross-correlogram; $i i$, CRP; $i i i$, RCRP). Event density in CRP and RCRP is indicated by the gray scale bar.

the-cross correlogram [(Fig. 4Di) the period of data and the unit (PGN1) are the same as in Figs. $2 A v i, 3 A v i]$, with CRP and RCRP (Fig. 4Dii, 4Diii) having an asynchronous pattern similar to those under free-run conditions (Fig. 4, compare $A, D$ ). 

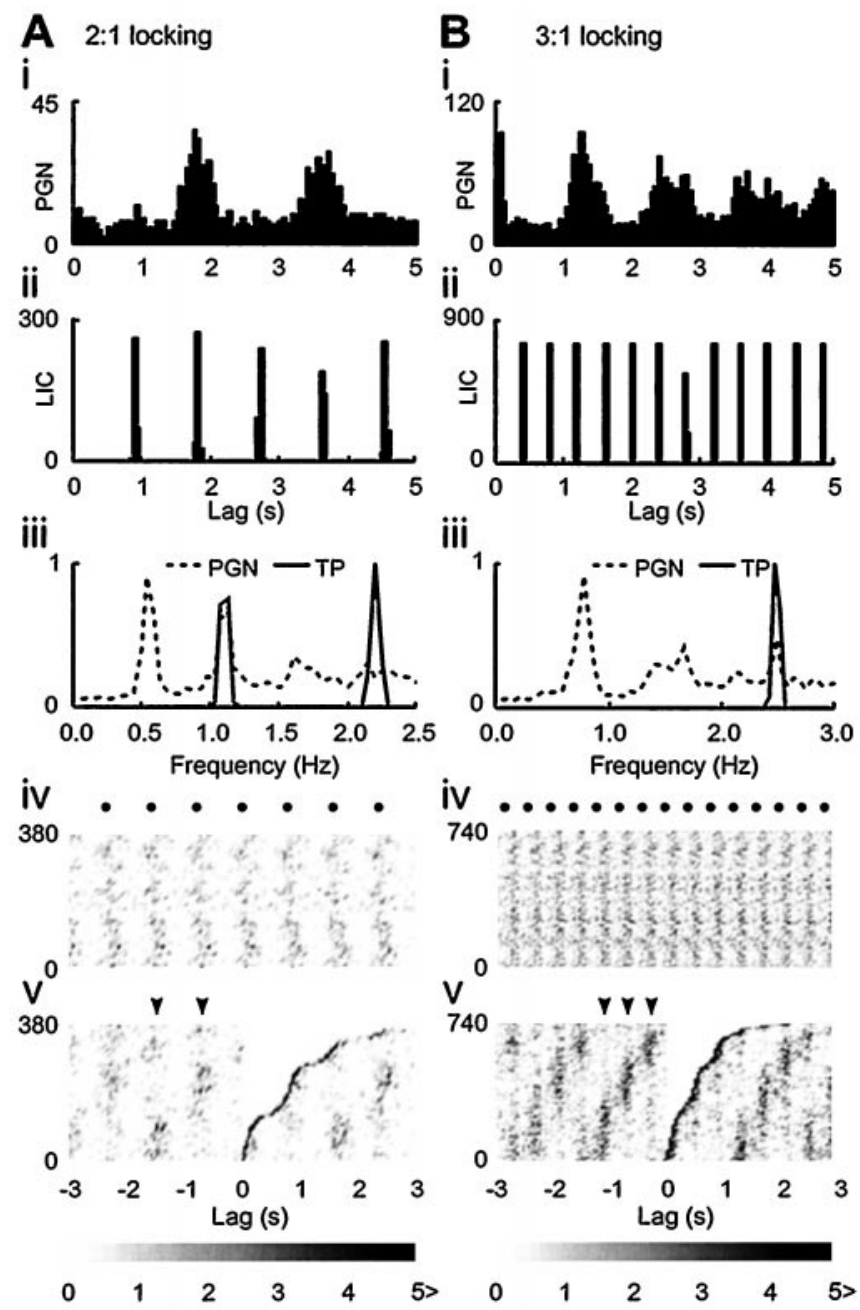

Figure 5. High-order rational frequency-lock. $A, 2: 1$ frequency-lock and $B, 3: 1$ frequency-lock. The respective frequency-locking ratio is suggested by the alignment of the first peak in the PGN autocorrelogram $(A i, B i)$ with the second and the third peak in the LIC autocorrelogram (Aii, Bii), respectively. A direct read-out of the 2:1 and the 3:1 frequency relationships is provided in their autospectra in which $f_{\text {LIC }}$ (LIC, solid line) coincides with the first harmonic frequency and the second harmonic frequency of PGN activity (dashed line), respectively (Aiii, Biii). Although the phase difference shows a stationary feature across time as revealed by the vertical bands in the CRP (Aiv, Biv; the relative positions of the LIC occurrences are indicated by filled circles), the RCRP demonstrates that the phase differences are grouped into two distinct clusters for 2:1 lock and three distinct clusters for 3:1 lock (arrowheads in $A v$ and $B v$, respectively). Event density in CRP and RCRP is indicated by the gray scale bar.

In some PGNs, when $f_{\text {LIC }}$ was high, high-order rational frequency-lock ratios, other than 1:1, were apparent. This is exemplified by $2: 1$ frequency-lock in Figure $5 A$. The alignment of the second peak in the LIC autocorrelogram and the first peak in the PGN autocorrelogram (Fig. 5Ai, Aii) and the coincidence of $f_{\text {LIC }}$ and the first harmonic frequency of the PGN dominant rhythm (Fig. 5Aiii) suggest a 2:1 relationship. The stationary phase difference across time (Fig. 5Aiv) was grouped into two distinct clusters (Fig. $5 A v$ ), a state consistent with 2:1 frequencylock. Although a systematic search for the whole range of other high-order frequency-lock states was not the purpose of this study, a 3:1 relationship was observed in one experiment when $f_{\text {LIC }}$ was increased to $2.41 \mathrm{~Hz}$ while a high tidal volume was maintained (Fig. 5Bi, 5Bii). The temporal stability and three- cluster grouping of the phase differences was apparent as shown in the CRP and RCRP (Fig. 5Biv, 5Bv).

In summary, high probability of 1:1 entrainment between LICs and single PGN activity could only be achieved when $f_{\text {LIC }}$ was close to the $f_{\text {INT }}$ of the PGN. As $f_{\text {LIC }}$ was moved away from PGN $f_{\text {INT }}$, a tight coupling between LICs and the PGN could not be maintained, but instead a state of relative coordination with PGN activity being "attracted" to LICs emerged intermittently. When $f_{\text {LIC }}$ was increased farther away from $\operatorname{PGN} f_{\text {INT }}$, an asynchronous pattern dominated their interaction. At these high $f_{\mathrm{LIC}} \mathrm{s}$ highorder rational frequency-lock other than 1:1 lock occurred in some PGNs. The change of frequency-dependent LIC-PGN coupling strength was reflected in the interaction between PGNs: high $\mathrm{PGN} \rightarrow$ PGN coherence resulted from tight LIC-PGN coupling. As PGN activity was dissociated from LICs, the coherence between PGNs dropped.

\section{The change of discharge patterns of single PGNs is not associated with a significant change of discharge rate}

To test whether the change of discharge patterns in response to $f_{\text {LIC }}$ variations induced a change in the neuronal excitability, the mean discharge rate (MDR) of the single PGNs across different $f_{\text {LIC }}$ was calculated and compared. Under free-run conditions, the median of the MDR of the single PGNs was $1.23 \mathrm{~Hz}$ (range, 0.75-2.71 Hz) ( $n=21$ PGNs from 12 animals). The comparison across the range of $f_{\text {LIC }}$ s tested was performed on the normalized MDR (normalized by the MDR during free-run) because of the wide variation of the MDR [see also Johnson and Gilbey (1996) and Chang et al. (1999b)]. Although a positive trend was observed in the normalized MDR as $f_{\text {LIC }}$ was increased, it was not significant ( $t$ test for zero slope of the regress line; $n=100$ cases for all the 21 PGNs across all the $f_{\text {LIC }} \mathrm{s}$ tested; $\left.p=0.055\right)$. This supports the notion that the neuronal excitability did not change significantly when $f_{\text {LIC }}$ was varied.

\section{Behavior emergent from the PGN population}

In the second type of experiment, we investigated the interaction between LICs and population PGN activity. The VCN discharge behavior in response to $f_{\text {LIC }}$ changes was similar in experiments with $\mathrm{Pa}_{\mathrm{CO}_{2}}$ clamp or without $\mathrm{Pa}_{\mathrm{CO}_{2}}$ clamp during hyperventilation. A typical example (without $\mathrm{Pa}_{\mathrm{CO}_{2}}$ clamp) showing the real time data and autospectra of the VCN and TP waves, and their coherence spectra, is shown in Figures $2 B$ and $3 B$, respectively. Under free-run conditions, the VCN autospectrum revealed a single broad peak (median peak frequency, $0.73 \mathrm{~Hz}$; interquartile range: $0.63-0.78$ ) (Fig. $2 \mathrm{Bi}$ ), and $\mathrm{LIC} \rightarrow \mathrm{VCN}$ coherence at $f_{\mathrm{LIC}}$ was low (Fig. $3 \mathrm{Bi}$ ). Single PGNs, recorded under the same conditions, exhibited a range of dominant frequencies and were weakly correlated or uncorrelated as described previously, suggesting that the population rhythm did not arise from mutual entrainment among the individual oscillators, but instead represented an average of the $f_{\text {INT }}$ range emergent from the whole oscillator pool (Christakos, 1986, 1994). Moving $f_{\text {LIC }}$ into this $f_{\text {INT }}$ range yielded a single narrow peak in the autospectra (0.59$0.78 \mathrm{~Hz}$ ) (Fig. 2Bii-iv) with high coherence (Fig. 3Bii-iv), suggesting that most PGNs in the population were 1:1 entrained to $f_{\text {LIC }}$. This finding was consistent with the observation at single PGN level. At high $f_{\text {LIC, }}$ however, in contrast with single PGN recordings, the sharp dominant peak at $f_{\text {LIC }}(1.00-1.35 \mathrm{~Hz})$ (Fig. $2 B v-v i)$ was preserved, although some PGNs must have escaped 1:1 entrainment, as suggested by the appearance of minor peaks 
in $f_{\text {INT }}$ range (Fig. $2 B v-v i$, arrows). At these high $f_{\text {LIC }} \mathrm{s}$, strong LIC-VCN coupling with high coherence was maintained $\left(f_{\text {LIC }}\right.$ : $1.00-1.35 \mathrm{~Hz}$ ) (Fig. $3 B v-v i$ ), although a moderate drop was observed as $f_{\text {LIC }}$ was moved farther away from the $f_{\text {INT }}$ range (Fig. 3, compare $B v, B v i)$.

In summary, like the strong LIC-PGN 1:1 coupling observed at single PGN level, when $f_{\text {LIC }}$ was close to the PGN $f_{\text {INT }}$ range, population PGN activity displayed prominent LIC-related rhythmical activity. However, in contrast to the findings in single PGNs, at high $f_{\text {LIC }} \mathrm{s}$, the dominant population rhythm still maintained 1:1 frequency-lock to LICs with only a moderate decline of LIC-population PGN coupling strength (see below).

\section{Comparison of the discharge behaviors of single PGN and population PGN activity}

The difference in single and population PGN behaviors in response to changes of $\mathrm{f}_{\text {LIC }}$ is summarized in Figure $6 A-C$. The $f_{\text {LIC }}$ range where the dominant $\mathrm{PGN}$ rhythm could maintain 1:1 lock to $f_{\text {LIC }}$ was wider for population PGN activity than for single PGN activity (Fig. $6 A$ ). When $f_{\text {LIC }}$ was increased stepwise, the dominant population rhythm could follow $f_{\text {LIC }}$ faithfully (dotted line), yet single PGN dominant frequencies remained close to their $f_{\text {INT }}$ range (Fig. $6 A$, shaded area). This driving frequencyrelated coupling depended on the frequency discrepancy between $f_{\text {LIC }}$ and $f_{\text {INT }}$. As illustrated in Figure $6 B$, although it is evident that population activity could maintain 1:1 frequency-lock (bottom dotted line) to LICs even when their frequency difference was up to $1.0 \mathrm{~Hz}$, this frequency difference zone for 1:1 lock was restricted to around $-0.2 \sim 0.2 \mathrm{~Hz}$ (shaded area) for single PGNs. In some cases, high-order rational frequency-lock between single PGNs and LICs such as 2:1 were apparent (Fig. 6B, top dotted line). In both cases, coherence dropped significantly as $f_{\text {LIC }}$ was moved away from $f_{\text {INT }}[t$ test for zero slope for the regression line, $p<0.001$ for both cases; single PGNs, filled circles and solid line; population PGNs, open circles and dotted line (Fig. 6C)]. However, the decline in coherence was moderate at population level compared with that at single unit level ( $t$ test for equal slope, $p<$ 0.001). The loss of entrainment of single PGNs to LICs was accompanied with reduction of coherence between two pairrecorded PGNs ( $t$ test for zero slope, $p<0.001$ ), and it is apparent that $\mathrm{PGN} \rightarrow \mathrm{PGN}$ coherence was higher when both units were 1:1 entrained to LICs [solid triangles, both units were entrained to LICs, vs open squares, at least one unit was not entrained to LICs (Fig. 6D)].

\section{DISCUSSION}

In this paper we reveal clear differences between the activity arising from a multiple oscillator population and that of its constituent single oscillators. It has been proposed that an autonomous central oscillator is responsible for the quasiperiodic activity recorded from a peripheral sympathetic nerve (Gebber, 1980). However, the findings from our previous study (Chang et al., 1999b) and the present work demonstrate that instead of a single oscillator driving VCN sympathetic nerve activity, the rhythm is generated by multiple oscillators with different intrinsic frequencies. The discharge behavior of the VCN activity should be considered as an emergent collective property of this oscillator population in which the synchronized discharges of even a small fraction of the population will produce a robust population rhythm (Elul, 1972). Our study shows that a stable output rhythm coupled to a periodic drive can be maintained across a wide driving frequency range in such a population, each constituent
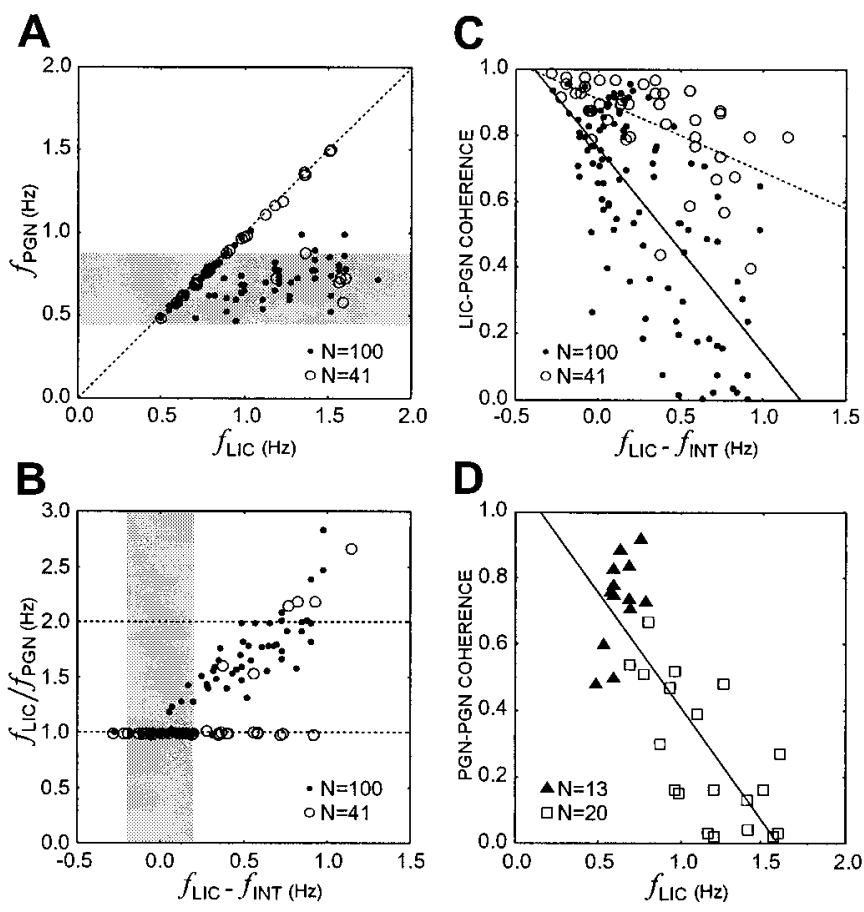

Figure 6. Summary scatter plots showing entrainment of single PGNs (filled circles) and population PGN (open circles) to LICs $(A-C)$ and the change of coherence between paired PGNs $(D)$. $A$, Dominant frequency $\left(f_{\mathrm{PGN}}\right)$ for single PGNs and population PGN plotted against $f_{\text {LIC }}$ illustrates the difference in distribution of $f_{\mathrm{PGN}} \mathrm{S}$ when $f_{\text {LIC }}$ is changed. Although $f_{\mathrm{PGN}}$ of single PGN activity remains close to the $f_{\mathrm{INT}}$ range (shaded area) when $f_{\text {LIC }}$ is increased, $f_{\mathrm{PGN}}$ of population activity can maintain 1:1 lock (dotted line) to LICs over a much wider range. $B$, The frequency ratio, $f_{\mathrm{LIC}} / f_{\mathrm{PGN}}$, plotted against the frequency difference, $f_{\mathrm{LIC}}-$ $f_{\text {INT }}$, provides evidence for frequency lock at 2:1 (top dotted line) as well as 1:1 (bottom dotted line). 1:1 entrainment for single PGNs is only possible when $f_{\text {LIC }}-f$ INT is less than $\sim 0.2 \mathrm{~Hz}$ (shaded area), whereas population $f_{\mathrm{PGN}}$ can follow $f_{\text {LIC }}$ faithfully over a broader range. $C, \mathrm{LIC} \rightarrow$ PGN coherence at $f_{\text {LIC }}$ plotted as a function of $f_{\text {LIC }-f \text { INT }}$ shows the decline of coherence at the single neuron (solid line) and the population (dotted line) levels ( $t$ test for zero slope of the regression lines; single PGNs: $p<0.001$, population PGNs: $p<0.001)$. The coherence drop is more moderate for population PGN activity than single PGN activity when $f_{\text {LIC }}$ is moved away from $f_{\text {INT }}(t$ test for equal slope of the regression lines, $p<0.001)$. $D$, The drop of LIC $\rightarrow$ PGN coherence as $f_{\text {LIC }}$ is increased is accompanied by decline of PGN $\rightarrow$ PGN coherence $(t$ test for zero slope of the regression line, $p<0.001)$. Higher coherence and therefore stronger coupling strength at $f_{\mathrm{LIC}}$ result when both units are 1:1 entrained to LICs (filled triangles, both PGNs are entrained, vs open squares, at least one PGN is not entrained).

oscillator exhibiting different LIC-PGN coupling dynamics as a result of the spread of $f_{\text {INT }} \mathrm{s}$. We suggest that this stable feature at the population level arises from the underlying linear and nonlinear interactions between the individual oscillators and the common drive.

\section{Rhythm generation from a population of weakly coupled or uncoupled oscillators}

Under free-run conditions in which each single PGN is "unlocked" so that it produces different intrinsic rhythms, a dominant rhythm still emerges from the population (Chang et al., 1999b). This finding is consistent with previous theoretical studies that predicted that a population of weakly coupled or uncoupled multiple oscillators is capable of generating a prominent rhythm if the $f_{\mathrm{INT}} \mathrm{S}$ are restricted to a narrow frequency range (Christakos, 1986, 1994). This provides an alternative to the model 
suggested in cardiac (Jalife, 1984; Michaels et al., 1986) or circadian (Liu et al., 1997) rhythm generation, in which mutual entrainment between constituent oscillators is necessary to explain the emergence of a population rhythm. We also show that at the population level there is a preservation of high coherence with LICs and a broader 1:1 frequency-locking zone compared with that of single PGNs. Our evidence suggests that this arises from three principal effects. The first is caused by the spread of intrinsic frequencies within the oscillator population. This allows a proportion of the pool to remain 1:1 entrained over a much wider driving frequency range than any single oscillator. However, at $f_{\text {LIC }}$ s above the $f_{\text {INT }}$ range of single PGNs, we suggest that a second phenomenon, relative coordination, contributes to the maintenance of high coherence. Relative coordination describes interactions in which oscillators are not synchronized but nevertheless exhibit intermittent and transient periods of phase-lock (von Holst, 1939; Kelso, 1995). The state of relative coordination disengages the single PGN from a state of tight 1:1 entrainment while maintaining weak coupling to LICs. We suggest that transient phase-lock will become a relatively stable feature at the population level, because at any given moment, there is a high probability that a subpopulation of PGNs will be phase-locked to LIC. Third, we propose that high-order rational frequencylocking also occurs. This would tend to channel single PGNs into a finite number of phase differences, which would prevent continuous phase-drift and contribute to the preservation of high coherence with LICs. The driving frequency-dependent interaction is also supported by the observation that while maintaining a constant drive strength (i.e., tidal volume), a transition of coupling states from tight 1:1 entrainment to complete asynchrony between PGNs and LICs can arise from a small increase of $f_{\text {LIC }}$. It must be emphasized that the mechanism underlying 1:1 frequency-lock between the dominant population rhythm and $f_{\text {LIC }}$ was different at different $f_{\text {LIC }}$ s. When $f_{\text {LIC }}$ was close to the center of the $f_{\mathrm{INT}}$ range of the PGN population, tight 1:1 entrainment of most PGNs probably accounted for the frequency-lock at the population level. However, when $f_{\text {LIC }}$ was moved away from the $f_{\text {INT }}$ range, with the resultant dissociation of $\mathrm{PGN}$ frequencies from $f_{\text {LIC }}$, the phase stabilizing effect observed in phenomena such as relative coordination and high-order rational frequency lock contributed to the maintenance of LIC-population PGN coupling.

In previous work in which a stable frequency-lock state in multifiber sympathetic activity was maintained across a wide driving frequency range, it was argued that this provided evidence indicating that sympathetic activity was not generated by an oscillator (Bachoo and Polosa, 1987). However, the findings from the present study provide evidence that oscillators do underlie sympathetic activity, and the phenomena of frequency-lock across a wide driving frequency can be explained by complex underlying dynamic processes at a single neuron level.

\section{Functional implication of rhythm genesis in a population composed of multiple weakly coupled or uncoupled oscillators}

Taken together, the driving frequency-dependent dynamics (of single oscillators) such as 1:1 entrainment, relative coordination, higher-order rational frequency-lock, and asynchrony are against a linear model in which frequency invariance is characteristic (Pavlidis, 1973; Bendat and Piersol, 1986). In contrast, they are consistent with nonlinear behaviors predicted from the theory of periodically driven biological oscillators (Pavlidis, 1973; Glass and
Mackey, 1988; Hilborn, 1994; Kelso, 1995). At the level of single oscillators, we suggest that the dynamic transition between the states of 1:1 entrainment, relative coordination, higher-order rational frequency-lock, and asynchrony results in large changes in the coupling strength with LICs, but at the level of the population of oscillators (with a spread of $f_{\mathrm{INT}} \mathrm{s}$ ), these LIC-PGN coupling fluctuations become smoothed and graded. This suggests that a population of weakly coupled or uncoupled oscillators is particularly suitable for producing patterned output activity and generating rapid and adaptive responses. If a system behaves like a single oscillator, it is expected that a moderate change of the driving parameters may cause a prominent change of the degree of synchrony between the driving force and the system owing to the underlying nonlinear coupling dynamics (Guevara et al., 1981; Petrillo and Glass, 1984). This is disadvantageous in a system in which the demand for stability is of paramount importance. On the other hand, it has been suggested that persistence of a global synchronous state of neurons has limited ability for information processing in the CNS (Singer, 1993). The disengagement of synchronous individual neurons is particularly important in the sympathetic nervous system controlling vascular resistance where the target organ behaves like an "integrator" with individual vascular smooth cells connected through gap junctions (Christ et al., 1996). A persistence of a synchronous state would most likely result in prolonged high vascular tone that may be harmful because coactivation of sympathetic nerve fibers innervating an artery, i.e., synchronization of PGN activity, has been shown to have a prominent effect on the contraction of the arterial smooth muscle (Hirst and Edwards, 1989). Our study shows that although retaining the capability to rapidly desynchronize neurons, the CVA PGNs can generate graded and smoothed synchronous activity coupled to the common drive, LIC-related activity. Although the influence of the strength of the periodic drive, i.e., the tidal volume, on the PGN oscillators was not investigated in the present study, our previous study has shown that the degree of synchronization between them can also be adjusted by varying the input strength (Chang et al., 1999b). In addition, the change of the PGN discharge behavior is not accompanied by a significant change of the discharge rate. This suggests that a wide range of PGN discharge patterns may be generated, without concomitant change of neuronal excitability, by changing the input parameters, either amplitude or frequency. The ability of the sympathetic nervous system to generate input-dependent patterning implies that temporal coding, as proposed in the cerebral cortex (Singer, 1996; Fetz, 1997), is used by this system to coordinate PGN activity, for example, to provide differential cardiovascular support to animals under stress (Vissing, 1997). Therefore, we propose that the principle of dynamic interactions of weakly coupled or uncoupled multiple oscillators in response to a driving force provides the system with metastability and allows it to respond in a graded dynamic manner to changes of input.

\section{REFERENCES}

Ayers JL, Selverston AI (1979) Monosynaptic entrainment of an endogenous pacemaker network: a cellular mechanism for von Holst's magnet effect. J Comp Physiol 129:5-17.

Bachoo M, Polosa C (1987) Properties of the inspiration-related activity of sympathetic preganglionic neurones of the cervical trunk in the cat. J Physiol (Lond) 385:545-564.

Bendat JS, Piersol AG (1986) Random data: analysis and measurement procedures. New York: Wiley.

Chang H-S (1999) Dynamic synchronization of sympathetic oscillators. $\mathrm{PhD}$ thesis, University College London, University of London. 
Chang H-S, Staras K, Gilbey MP (1999a) Complex dynamics of periodically driven sympathetic oscillators. Soc Neurosci Abstr 25:759.7.

Chang H-S, Staras K, Smith JE, Gilbey MP (1999b) Sympathetic neuronal oscillators are capable of dynamic synchronization. J Neurosci 19:3183-3197.

Christ GJ, Spray DC, el-Sabban M, Moore LK, Brink PR (1996) Gap junctions in vascular tissues: evaluating the role of intercellular communication in the modulation of vasomotor tone. Circ Res 79:631-646.

Christakos CN (1986) The mathematical basis of population rhythms in nervous and neuromuscular systems. Int J Neurosci 29:103-107.

Christakos CN (1994) Analysis of synchrony (correlations) in neural populations by means of unit-to-aggregate coherence computations. Neuroscience 58:43-57.

DeHaan RL, Hirakow R (1972) Synchronization of pulsation rates in isolated cardiac myocytes. Exp Cell Res 70:214-220.

Elul R (1972) Randomness and synchrony in the generation of the electroencephalogram. In: Synchronization of EEG activity in epilepsies (Petsche H, Brazier MA, eds), pp 59-77. New York: Springer.

Fetz EE (1997) Temporal coding in neural populations? Science 278:1901-1902.

Gebber GL (1980) Central oscillators responsible for sympathetic nerve discharge. Am J Physiol 239:H143-H155.

Gilbey MP, Spyer KM (1997) Cardiorespiratory regulation. In: Neural control of the respiratory muscles (Miller AD, Bianchi AL, Bishop BP, eds), pp 259-269. Boca Raton, FL: CRC.

Glantz SA (1996) Primer of biostatistics. New York: McGraw-Hill.

Glass L, Mackey MC (1988) From clocks to chaos: the rhythms of life. Princeton: Princeton UP.

Guevara MR, Glass L, Shrier A (1981) Phase locking, period-doubling bifurcations, and irregular dynamics in periodically stimulated cardiac cells. Science 214:1350-1353.

Herzog ED, Takahashi JS, Block GD (1998) Clock controls circadian period in isolated suprachiasmatic nucleus neurons. Nat Neurosci 1:708-713.

Hilborn RC (1994) Chaos and nonlinear dynamics. New York: Oxford UP.

Hirst GD, Edwards FR (1989) Sympathetic neuroeffector transmission in arteries and arterioles. Physiol Rev 69:546-605.

Jalife J (1984) Mutual entrainment and electrical coupling as mechanisms for synchronous firing of rabbit sino-atrial pace-maker cells. J Physiol (Lond) 356:221-243.

Johnson CD, Gilbey MP (1994) Sympathetic activity recorded from the rat caudal ventral artery in vivo. J Physiol (Lond) 476:437-442.

Johnson CD, Gilbey MP (1996) On the dominant rhythm in the discharges of single postganglionic sympathetic neurones innervating the rat tail artery. J Physiol (Lond) 497:241-259.

Kelso JAS (1995) Dynamic patterns: the self-organization of brain and behavior. Cambridge, MA: MIT.

Lipski J, Coote JH, Trzebski A (1977) Temporal patterns of antidromic invasion latencies of sympathetic preganglionic neurons related to cen- tral inspiratory activity and pulmonary stretch receptor reflex. Brain Res 135:162-166.

Liu C, Weaver DR, Strogatz SH, Reppert SM (1997) Cellular construction of a circadian clock: period determination in the suprachiasmatic nuclei. Cell 91:855-860.

Marshall JM (1994) Peripheral chemoreceptors and cardiovascular regulation. Physiol Rev 74:543-594.

Michaels DC, Matyas EP, Jalife J (1986) Dynamic interactions and mutual synchronization of sinoatrial node pacemaker cells. A mathematical model. Circ Res 58:706-720.

Pavlidis T (1973) Biological oscillators: their mathematical analysis. New York: Academic.

Perkel DH, Gerstein GL, Moore GP (1967a) Neuronal spike trains and stochastic point processes. I. The single spike train. Biophys J 7:391-418.

Perkel DH, Gerstein GL, Moore GP (1967b) Neuronal spike trains and stochastic point processes. II Simultaneous spike train. Biophys J 7:419-440.

Petrillo GA, Glass L (1984) A theory for phase locking of respiration in cats to a mechanical ventilator. Am J Physiol 246:311-320.

Rosenberg JR, Amjad AM, Breeze P, Brillinger DR, Halliday DM (1989) The Fourier approach to the identification of functional coupling between neuronal spike trains. Prog Biophys Mol Biol 53:1-31.

Rosenblum LD, Turvey MT (1988) Maintenace tendency in coordinated rhythmic movements: relative fluctuations and phase. Neuroscience 27:289-300.

Sano T, Sawanobori T, Adaniya H (1978) Mechanism of rhythm determination among pacemaker cells of the mammalian sinus node. Am J Physiol 235:H379-H384.

Singer W (1993) Synchronization of cortical activity and its putative role in information processing and learning. Annu Rev Physiol 55:349-374.

Singer W (1996) Putative functions of temporal correlations in neocortical processing. In: Large-scale neuronal theories of the brain (Koch C, Davis JL, eds), pp 201-237. Cambridge, MA: MIT.

Sittiracha T, McLachlan EM, Bell C (1987) The innervation of the caudal artery of the rat. Neuroscience 21:647-659.

Smith JE, Jansen AS, Gilbey MP, Loewy AD (1998) CNS cell groups projecting to sympathetic outflow of tail artery: neural circuits involved in heat loss in the rat. Brain Res 786:153-164.

Vissing SF (1997) Differential activation of sympathetic discharge to skin and skeletal muscle in humans. Acta Physiol Scand [Suppl] 639:1-32.

von Holst E (1939) The behavioural physiology of animals and man: the selected papers of Erich von Holst (Martin R, translator), pp 33-135. London: Methuen.

Welsh DK, Logothetis DE, Meister M, Reppert SM (1995) Individual neurons dissociated from rat suprachiasmatic nucleus express independently phased circadian firing rhythms. Neuron 14:697-706.

Winfree AT (1980) The geometry of biological time. New York: Springer. 